\title{
Diacronie
}

Studi di Storia Contemporanea

$\mathrm{N}^{\circ} 28,4 \mid 2016$

La voce del silenzio

\section{Le reti di spionaggio e sabotaggio nazifasciste nell'Italia occupata dagli Alleati (1943-1945)}

\section{Nicola Tonietto}

\section{(C) OpenEdition}

\section{Journals}

\section{Edizione digitale}

URL: http://journals.openedition.org/diacronie/4718

DOI: $10.4000 /$ diacronie.4718

ISSN: 2038-0925

\section{Editore}

Association culturelle Diacronie

\section{Notizia bibliografica digitale}

Nicola Tonietto, «Le reti di spionaggio e sabotaggio nazifasciste nell'Italia occupata dagli Alleati (1943-1945) », Diacronie [Online], N 28, 4 | 2016, documento 5, Messo online il 29 décembre 2016 consultato il 30 avril 2019. URL : http://journals.openedition.org/diacronie/4718; DOI : 10.4000/ diacronie. 4718 


\section{Diacronie}

\section{Le reti di spionaggio e sabotaggio nazifasciste nell'Italia occupata dagli Alleati (1943-1945)}

\section{Nicola TONIETTO *}

Questo lavoro si prefigge lo scopo di descrivere le reti di spionaggio e di sabotaggio, le missioni e gli agenti dei servizi segreti tedeschi e della RSI inviati nell'Italia centromeridionale, tra il 1943 e il 1945, con l'obiettivo di contrastare l'avanzata Alleata nella Penisola. I due servizi segreti tedeschi, l'Abwehr e il Sicherheitsdienst, riorganizzati dopo l'Armistizio, reclutarono agenti italiani (provenienti dalla Milizia o dalle neonate Brigate Nere ma anche dalla Decima Mas di Borghese) per operazioni di spionaggio e sabotaggio oltre le linee Alleate. Anche gli stessi fascisti, tramite il neonato SID ed altri gruppi come quello del Principe Pignatelli o l'Ufficio Pucci-Del Massa, tentarono di pianificare reti spionistiche ma anche di porre le basi per la sopravvivenza del fascismo all'indomani della fine della guerra.

\section{Introduzione}

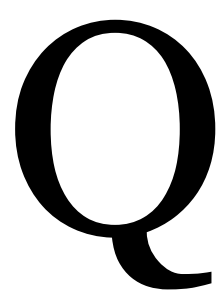

uesto saggio si prefigge lo scopo di descrivere le reti di spionaggio e di sabotaggio, le missioni e gli agenti dei servizi segreti tedeschi e della Repubblica Sociale Italiana inviati nell'Italia centro-meridionale, tra il 1943 e il 1945, con l'obiettivo di contrastare l'avanzata Alleata nella Penisola. La ricerca si basa principalmente sull'analisi dei documenti italiani provenienti dall'Archivio del Servizio Informazioni Militare (SIM), il servizio segreto dell'esercito, e Alleati (Allied Force Headquarters, $15^{\text {th }}$ Army Group), vista anche la quasi assenza di studi sull'argomento. Se infatti la bibliografia riguardante i rapporti tra lo spionaggio alleato e la resistenza italiana è cospicua, ed è stata incrementata in particolare negli ultimi anni, poco è stato scritto sull'attività di 
constrospionaggio e controsabotaggio, ovvero sul contrasto all'attività di intelligence Nazista e Fascista nell'Italia controllata dagli Alleati ${ }^{1}$. Allo stesso modo risultano quasi inesistenti gli studi sulle organizzazioni spionistiche del Reich e della Repubblica Sociale e sulle operazioni portate avanti ai danni del Regno del Sud².

L'analisi delle organizzazioni di intelligence nazifasciste si inserisce nel contesto dello studio delle strutture di occupazione messe in atto dalla Germania nazista all'indomani dell'armistizio. Allo stesso modo, può risultare un utile filone di ricerca per la storia della Repubblica Sociale Italiana e della sua situazione di «alleato occupato»3. I servizi di Salò infatti, e coloro i quali decisero di far parte di organizzazioni spionistiche per tentare di contrastare l'avanzata alleata, furono costretti, così come le molteplici organizzazioni del fascismo repubblicano, a vedere frustrate le proprie velleità di autonomia nei confronti delle decisioni prese unilateralmente dell'alleato tedesco 4 .

Nel nostro Paese operarono entrambi i servizi segreti tedeschi, l'Abwehr e il Sicherheitsdienst, i quali reclutarono in massa agenti italiani (provenienti dalla Milizia

${ }^{1}$ Per citare solo alcuni tra i lavori più recenti sul rapporto tra Alleati e Resistenza: MILLAN, Matteo, Guerra di servizi: tra Italia e Svizzera la rete informativa della Resistenza, Padova, Il Poligrafo, 2009; BERRETTINI, Mireno, La Resistenza italiana e lo Special Operation Executive britannico (1943-1945), Firenze, Le lettere, 2014; SAPIO, Michaela, Spie in guerra: l'intelligence americana dalla caduta di Mussolini alla Liberazione (1943-1945), Milano, Mursia, 2015. L'unico lavoro specifico sul Servizio Informazioni Militare è quello di CONTI, Giuseppe, Una guerra segreta. Il SIM nel secondo conflitto mondiale, Bologna, Il Mulino, 2009, che però si concentra quasi esclusivamente 1939-1943. Il lavoro del SIM a fianco degli Alleati è appena accennato nelle pagine conclusive del volume.

2 Per quanto riguarda l'attività dei servizi nazifascisti in Italia si possono annoverare: COLLOTTI, Enzo, «Documenti sull'attività del Sicherheitsdienst nell'Italia occupata», il Il Movimento di liberazione in Italia, 71, 2/1963, pp. 38-77; GENTILE, Carlo, I servizi segreti tedeschi in Italia 1943-1945, in FERRARI, Paolo, MASSIGNANI, Alessandro (a cura di), Conoscere il nemico. Apparati di intelligence e modelli culturali nella storia contemporanea, Milano, Franco Angeli, 2010, pp. 459-495; PALA, Elena, «Il Servizio Informazioni Difesa della Repubblica sociale italiana. Il caso del nucleo di controspionaggio di Brescia», in Annale dell'Archivio della Resistenza bresciana e dell'età contemporanea, 5, 2009, pp. 159-185. Il volume di FRANZINELLI, Mimmo, Guerra di spie. I servizi segreti fascisti, nazisti e alleati (1939-1943), Milano, Mondadori, 2006, come si evince dal titolo, non si occupa del periodo preso in considerazione dal presente saggio.

3 KLINKHAMMER, Lutz, L'occupazione tedesca in Italia (1943-1945), Torino, Bollati Borighieri, 1993, p. 5. Per una analisi sulle linee di ricerca riguardanti la Repubblica Sociale Italiana vedi ROVATTI, Toni, "Linee di ricerca sulla Repubblica Sociale Italiana», in Studi Storici , 55, 1/2014, pp. 287-299.

4 Esemplificativa è, ad esempio, la questione della creazione dell'esercito della Repubblica Sociale, ripetutamente ostacolata dai tedeschi. Vedi GANAPINI, Luigi, La repubblica delle camicie nere, Milano, Garzanti, 1999, pp. 70-85, ma anche FIORAVANZO, Monica, Mussolini e Hitler. La Repubblica sociale sotto il Terzo Reich, Roma, Donzelli, 2009, pp. 135-155. Maggiore autonomia venne invece concessa alla Guardia Nazionale Repubblicana, nata come corpo di polizia militare che accorpava ex Carabinieri, uomini della Miliza, ufficiali di Polizia e membri della Polizia Africa Italiana (PAI) e in seguito alle Brigate Nere, le milizie del Partito fascista repubblicano. Vedi GANAPINI, Luigi, op.cit, pp. 30-46; GAGLIANI, Dianella, Brigate nere. Mussolini e la militarizzazione del Partito fascista repubblicano, Torino, Bollati Boringhieri, 1999. 
fascista o dalle neonate Brigate Nere ma anche dalla Decima Mas di Borghese) per operazioni di spionaggio e sabotaggio oltre le linee Alleate. Gli stessi fascisti, inoltre, tentarono di pianificare autonomamente delle reti spionistiche e collateralmente provare a porre le basi per la sopravvivenza del fascismo all'indomani della fine della guerra.

\section{L'intelligence nazifascista in Italia}

La presenza in Italia dei servizi segreti del Reich risaliva al 1939, quando iniziò ad operare la sezione dedicata allo spionaggio estero del Sicherheitsdienst (chiamata Ausland-SD o Amt VI), ovvero l'agenzia di spionaggio civile del Terzo Reich inglobata nel Reichssicherheitshauptamt (RSHA, Ufficio per la sicurezza dello Stato) ${ }^{5}$. Un documento del controspionaggio italiano definiva l'azione del SD nel nostro Paese «come un controspionaggio politico svolto con modalità offensive e di penetrazione» ${ }^{6}$. Contemporaneamente era presente anche l'Abwehr, il servizio informativo della Wehrmacht, la cui centrale romana era diretta dal colonnello Otto Hellferich. Fino all'8 settembre 1943, l'Abwehr in Italia si occupava principalmente di controspionaggio (Terzo Abwehr o Abwehr III) oltre che del reclutamento di agenti da inviare in missioni in Grecia o nel Medio Oriente?.

La situazione per entrambi i servizi cambiò radicalmente quando venne reso noto l'Armistizio tra Italia e gli Alleati. Già nell'agosto del 1943, tuttavia, il generale Karl Wolff era stato designato come "Comandante supremo della polizia e delle SS in Italia" il quale da Monaco aveva iniziato ad organizzare la futura struttura delle SS in Italia ${ }^{8}$. Nonostante le rassicurazioni di Badoglio sul proseguimento della guerra a fianco del Reich, la destituzione di Mussolini aveva minato significativamente la fiducia dei tedeschi nei confronti dell'ormai ex alleato. Pertanto, nei mesi di luglio e agosto, le truppe tedesche non vennero più inviate verso il Sud e la Sicilia, dove gli Alleati avevano aperto il nuovo fronte europeo, ma iniziarono ad occupare posizioni strategiche anche in Italia settentrionale. I porti di Genova, La Spezia, Trieste, ma

\footnotetext{
5 GENTILE, Carlo, I servizi segreti tedeschi in Italia 1943-1945, p. 462.

6 The National Archives (d'ora in avanti TNA), WO 204/12293, History of German intelligence organization in Italy 1943-1945, rel. s.d., "Appunti sull'organizzazione informativa tedesca", p. 25 .

7 Archivio Ufficio Storico Stato Maggiore dell'Esercito, Fondo Servizio Informazioni Militare (d'ora in avanti AUSSME, SIM), b. 66, f. 1-1-1 1943 Organizzazione informativa tedesca in Italia, rel. del 19 ottobre 1943 "Appunti sull'organizzazione informativa tedesca in Italia e su alcune persone maggiormente in vista", pp. 1-2.

8 KLINKHAMMER, Lutz, op.cit., pp. 84-86.
} 
anche la capitale vennero letteralmente accerchiati ${ }^{9}$. Al contrario del Regio esercito dunque, l'8 settembre non colse impreparati né la Wehrmacht né le SS, le cui forze occuparono il territorio italiano senza grandi difficoltà ${ }^{10}$.

Anche l'Abwehr, il servizio dell'esercito, non esitò ad inviare personale che potesse organizzare anche in Italia la Prima e la Seconda sezione, rispettivamente avente compiti di spionaggio e sabotaggio. Allargò inoltre il suo raggio d'azione, espandendo in primo luogo la rete già presente nell'Italia settentrionale con sede a Milano, capeggiata dal maggiore Hans Werner, e istituendo alcuni sottocentri a Genova, Torino e, per il controllo della frontiera italo-svizzera, a Domodossola e Chiasso ${ }^{11}$. Nelle due zone di operazioni Adriatisches Kustenland e Alpenvorland, direttamente annesse al Reich, vennero fondati tre sottocentri a Trento, Bolzano e Trieste che svolgevano, oltre ad attività di controspionaggio, anche propaganda a favore della Germania ${ }^{12}$. I due nuovi settori aggiunti dovettero inoltre istituire delle scuole per addestrare i loro agenti. Il Primo Abwehr aveva scelto Firenze e Milano, la prima per l'esercito e la marina e la seconda per l'aviazione, come sedi per il reclutamento e l'addestramento di agenti informatori da inviare nel territorio occupato dagli Alleati. Al personale veniva insegnato come riconoscere gli stemmi e le armi alleate, oltre che individuare le loro navi mercantili e da guerra, gli aerei e aeroporti, nonché segnalarne la posizione. Al termine dell'addestramento, gli agenti venivano inviati in coppia, un informatore ed un radio-telegrafista, aventi rispettivamente il compito di raccogliere notizie e di trasmetterle ${ }^{13}$. Essi dovevano attraversare le linee nemiche a piedi oppure venivano aviolanciati o fatti sbarcare sulla costa da imbarcazioni o sommergibili. Erano forniti di sistemi di riconoscimento, in particolare il Servizio provvedeva a dotarli di un fazzoletto bianco «con al centro, riportata con inchiostro simpatico la dicitura che l'agente fa[ceva] parte del servizio tedesco di informazione» ${ }^{14}$. Il Secondo Abwehr, capeggiato dal conte di origine sudtirolese Thun von Hohenstein, aveva invece istituito inizialmente due scuole per sabotatori nell'isola di S.Andrea a Venezia e a Perugia ${ }^{15}$. I corsi duravano solitamente dalle 6 alle 7 settimane e ciascun corso era seguito da un

\footnotetext{
9 GENTILE, Carlo, I crimini di guerra tedeschi in Italia, Torino, Einaudi, 2015, p. 38.

10 Ibidem, pp. 40-46. Per le conseguenze dell'armistizio vedi AGA ROSSI, Elena, Una nazione allo sbando. L'armistizio italiano del settembre 1943, Bologna, Il Mulino, 2003.

11 AUSSME, SIM, b. 334, f. 1-1-2/6 1945 Studio sullorganizzazione del S.I. tedesco e repubblicano in Italia, rel. del 25 gennaio 1945 "Memoria circa l'organizzazione dei servizi informativi tedeschi”, n. prot. 1229, pp. 6-7.

12 Ibidem, p. 7.

13 Ibidem, pp. 7-8.

14 Ibidem, p. 12.

15 Ibidem, pp. 16-17.
} 
numero limitato di agenti ${ }^{16}$. L'addestramento nell'uso degli esplosivi era meticoloso: le reclute dovevano fare molta pratica in allenamento prima che venisse permesso loro di mettere in atto le capacità acquisite contro il nemico. Alla fine dell'addestramento gli agenti venivano paracadutati oltre le linee nemiche oppure abbandonati in caso di ritirata. Venivano impiegati individualmente o in gruppi e nel secondo caso, il capo del gruppo aveva a disposizione un radio-telegrafista. Era inoltre l'unico a conoscere dove poter rinvenire l'esplosivo nascosto dall'esercito tedesco in ritirata ${ }^{17}$. Le loro azioni avrebbero dovuto essere dirette principalmente contro linee ferroviarie, treni, magazzini, depositi di armi e carburante, centraline elettriche e telefoniche ${ }^{18}$.

Agli agenti dell'Abwehr venivano assegnati un nome in codice (che differiva a seconda dell'unità di appartenenza), un nome e una storia di copertura ${ }^{19}$. Solitamente, come riportato dall'intelligence italiana, gli agenti avrebbero dovuto raccontare di essere scappati dal Nord per evitare di essere obbligati a lavorare nell'Organizzazione Todt ${ }^{20}$. Erano inoltre provvisti di documenti falsi (carta d’identità e carta di appartenenza alla Todt) che avrebbero aiutato a supportare la loro storia se fossero stati catturati $^{21}$. Prima della partenza veniva anticipata una parte del compenso, il rimanente invece era riscosso nel momento del ritorno alla loro unità, una volta completata la missione ${ }^{22}$.

Secondo lo stesso SIM, nonostante l'addestramento di alto livello, i risultati ottenuti dal Secondo Abwehr erano però nettamente inferiori se comparati all'azione degli agenti del SD.

Anche il servizio segreto delle SS aveva dovuto modificare ed incrementare la propria struttura in seguito all'apertura del fronte italiano. Innanzitutto il generale Wilhelm Harster, giurista bavarese, venne designato come comandante della Sicherheitspolizei (Sipo, Polizia di sicurezza) e del SD in Italia con sede a Verona, ovvero l'organismo che coordinava l'attività della Gestapo, della Kripo (Polizia

\footnotetext{
16 Ibidem, p. 16.

17 Ibidem.

18 AUSSME, SIM, b. 334, f. 1-1-2/6 1945 Studio sull'organizzazione del S.I. tedesco e repubblicano in Italia, rel. del 20 febbraio 1945 "Studio orientativo sull'organizzazione, metodi e attività dei servizi informativi tedesco e repubblicano in Italia”, n. prot. segreto, p. 16.

19 AUSSME, SIM, b. 334, f. 1-1-2/6 1945 Studio sull'organizzazione del S.I. tedesco e repubblicano in Italia, rel. del 25 gennaio 1945 "Memoria circa l'organizzazione dei servizi informativi tedeschi”, n. prot. 1229, pp. 13-14.

20 AUSSME, SIM, b. 334, f. 1-1-2/6 1945 Studio sull'organizzazione del S.I. tedesco e repubblicano in Italia, rel. del 20 febbraio 1945 "Studio orientativo sull'organizzazione, metodi e attività dei servizi informativi tedesco e repubblicano in Italia”, n. prot. segreto, pp. 22-23.

${ }^{21}$ Ibidem, p. 23.

22 Ibidem, p. 17.
} 
criminale) e del SD, diviso tra Inland e Ausland (Interno ed Estero) ${ }^{23}$. Pertanto, anche le altre due polizie iniziarono ad operare nel territorio italiano e, secondo i Servizi italiani, erano dirette inizialmente da Eugen Dollmann e Herbert Kappler24. L'Ausland$S D$, diretto da Karl Hass, prima a Roma e poi a Verona, iniziò ad interessarsi anche all'attività di sabotaggio sia materiale che morale, la cosiddetta attività di "quinta colonna", andando a scontrarsi e intralciarsi con il Secondo Abwehr ${ }^{25}$. Per operazioni di spionaggio e sabotaggio il Servizio reclutava ed addestrava agenti in una scuola situata a Bologna. Un'altra scuola per sabotatori era stata predisposta a Roma mentre inizialmente gli agenti venivano inviati per l'addestramento a L'Aia in Olanda ${ }^{26}$. Durante i corsi, di circa un mese, i sabotatori venivano istruiti nell'impiego di esplosivi, nell'uso di armi tedesche e alleate, nella tattica del sabotaggio e nell'uso di automezzi ${ }^{27}$. Agli agenti del SD veniva consegnato, oltre che materiale esplosivo, del cianuro per potersi suicidare in caso di cattura ${ }^{28}$.

Ma chi erano questi agenti e da dove venivano? Tra la seconda metà del 1943 e i primi mesi del 1944, Abwehr e Sicherheitsdienst arruolarono principalmente tedeschi e sudtirolesi che conoscessero la lingua italiana, in modo tale da permettere loro di operare più facilmente alle spalle della linea del fronte. Verso la fine del 1944, come ci informa il contro-spionaggio italiano, la situazione era molto differente. Tra gli agenti nemici arrestati sia dal SIM che dagli Alleati nel corso del 1944 infatti, più dell'85\% erano italiani ${ }^{29}$. Lo stesso report ci illustra che tra costoro figuravano disertori, persone ricercate per crimini comuni, ma la maggior parte erano fascisti fanatici ${ }^{30}$. L'iniziale difficoltà per i Servizi tedeschi nell'arruolare personale in loco, aveva portato ad ingaggiare persone poco affidabili che avevano accettato di diventare agenti solamente

23 Wilhelm Harster aveva ricoperto lo stesso incarico in Olanda, dove nel dopoguerra venne processato e condannato per la sua attività in quel paese, in particolare per quanto riguardava la deportazione degli ebrei. Fu anche un alto funzionario ministeriale bavarese fino agli anni Sessanta. GENTILE, Carlo, I servizi segreti tedeschi in Italia 1943-1945, p. 468.

24 TNA, WO 204/12293, History of German intelligence organization in Italy 1943-1945, rel. s.d., "Appunti sull'organizzazione informativa tedesca in Italia", p. 24. Per quanto riguarda il ruolo di Dollmann in Italia, secondo Gentile, tuttavia, egli non fu altro che un «esperto di pubbliche relazioni delle SS in Italia», dove era presente già dal 1937. Vedi GENTILE, Carlo, I servizi segreti tedeschi in Italia 1943-1945, p. 465.

25 TNA, WO 204/12293, History of German intelligence organization in Italy 1943-1945, rel. s.d., "Appunti sull'organizzazione informativa tedesca in Italia", p. 25. Si tratta del noto Karl Hass, tra i responsabili, assieme a Kappler ed Erich Priebke dell'eccidio delle Fosse Ardeatine.

${ }_{26}$ AUSSME, SIM, b. 334, f. 1-1-2/6 1945 Studio sull'organizzazione del S.I. tedesco e repubblicano in Italia, rel. del 20 febbraio 1945 "Studio orientativo sull'organizzazione, metodi e attività dei servizi informativi tedesco e repubblicano in Italia”, n. prot. segreto, p. 25.

27 Ibidem.

${ }^{28}$ Ibidem, p. 26.

29 AUSSME, SIM, b. 150, f. 1-18-85 Relazione annuale sull'attività di C.S., rel. del 17 dicembre 1944 “Relazione sull'attività svolta dai Centri e Sezioni C.S. nell'anno 1944", p. 3.

3o Ibidem. 
per la generosa remunerazione che veniva loro garantita ${ }^{31}$. Tuttavia, nel corso del 1944 , la situazione cambiò quando i Servizi tedeschi riuscirono ad arruolare agenti provenienti da organizzazioni fasciste che potessero essere più affidabili come per esempio la Decima MAS di Junio Valerio Borghese, la Guardia nazionale repubblicana o la banda Koch ${ }^{32}$.

\section{Le reti di intelligence della Repubblica Sociale}

Già all'indomani dell'8 settembre i tedeschi si premurarono di annunciare agli italiani la proclamazione di un governo nazionale fascista nonostante Mussolini fosse ancora prigioniero33. Con la liberazione del Duce da parte del commando guidato da Otto Skorzeny, fu finalmente possibile fare insediare il nuovo governo fascista. Il 23 settembre 1943 nasceva, dunque, la Repubblica Sociale Italiana, anche se il nome divenne ufficialmente tale solo il $1^{\text {o }}$ dicembre 34 .

Secondo un'informativa conservata nell'archivio della divisione Servizi informativi e speciali del Ministero dell'Interno, «[n]el mese di ottobre del 1943 per ordine di Mussolini, Alessandro Pavolini formò dei gruppi di elementi fascisti di provata fede per creare un movimento di rinascita del fascismo nell'Italia meridionale»35. Fu quindi fin dai primi giorni del neonato governo che il Duce si preoccupò dell'organizzazione di "quinte colonne" fasciste nel territorio occupato dagli Alletati. A dirigere l'ufficio creato per tale scopo fu chiamato Puccio Pucci, identificato come «ex ufficiale dei moschettieri di Mussolini e Capo di stato maggiore generale delle Brigate Nere»36. L’organizzazione,

${ }^{31}$ Ibidem, p. 2, ma anche TNA WO 204/12987 German intelligence service vol.1, rel. del 15 Aprile 1944 "Enemy intelligence service in Italy", pp. 3-4.

${ }^{22}$ La Decima, nonostante appartenesse formalmente alla Marina della Repubblica Sociale Italiana, era un reparto militare indipendente e direttamente alleato ai nazisti tramite un accordo siglato dallo stesso Borghese con il capitano di vascello della Kriegsmarine Max Berninghaus. La banda Koch era invece uno corpo speciale di Polizia con a capo Pietro Koch incaricata di dare la caccia ai partigiani sotto la protezione di Kappler. Per approfondire vedi: GANAPINI, Luigi, op. cit., pp. 60-70; GRINER, Massimiliano, La «Banda Koch» reparto speciale di polizia (1943-1944), Torino, Bollati Boringhieri, 2000.

33 KLINKHAMMER, Lutz, op. cit., p. 49.

34 OSTI GUERRAZZI, Amedeo, Storia della Repubblica Sociale Italiana, Roma, Carocci, 2012, p. 87.

35 Archivio Centrale dello Stato, Direzione Generale di Pubblica Sicurezza, Servizio informazioni speciali-Sezione II (d'ora in avanti ACS, DGPS, SIS-II), b. 38, f. HP40 Penne stilografiche esplosive, relazione senza data, senza protocollo, p. 1.

36 Ibidem. Di famiglia nobile fiorentina, Puccio Pucci fu dapprima segretario del CONI dal 1939 al 1943 e in seguito capo di gabinetto di Alessandro Pavolini, oltre che capo del Servizio Informativo Fascista Repubblicano o Ufficio PdM. Al contrario di quanto citato nella nota informativa, non risulta invece che abbia ricoperto l'incarico di Capo di stato maggiore delle Brigate nere, affidato in primo luogo a Giovan Battista Riggio e in seguito a Edoardo Facdouelle. Vedi GAGLIANI, Dianella, op. cit., p. 154. 
tuttavia, indicata nelle carte del controspionaggio alleato con il nome di Servizio Informativo Fascista Repubblicano, non svolse attività particolari fino all'arrivo dello scrittore Aniceto del Massa, il quale negli ultimi mesi del 1944, su incarico di Pavolini, affiancò Pucci nella direzione del Servizio37. Ufficio che prese pertanto il nome di PdM dalle iniziali dei cognomi dei due direttori. Non era, d'altronde, la sola iniziativa che cercasse di creare dall'alto una resistenza fascista nel Sud Italia. Secondo Alfredo Cucco, tra i membri fondatori del Movimento Sociale Italiano (MSI) ed ex vice segretario del Partito Nazionale Fascista (PNF) nel 1943, Carlo Scorza, ultimo segretario del PNF, aveva incaricato il principe Valerio Pignatelli di Cerchiara, nella primavera del 1943, di dare vita ad una organizzazione di volontari che avrebbero dovuto agire alle spalle degli invasori con azioni di guerriglia e sabotaggi ${ }^{38}$. Le cosiddette Guardie ai Labari, questo il nome del gruppo, sarebbero state tuttavia liquidate dallo stesso Mussolini nella fase embrionale del progetto39.

Contemporaneamente all'instaurazione dell'Ufficio PdM, nell'ottobre 1943 veniva creato il servizio informativo della Repubblica Sociale Italiana, nominato Servizio Informazioni Difensivo (SID), e dipendente del Ministero delle Forze Armate della Repubblica di Salò ${ }^{\circ}$. Il Servizio, che aveva sede a Volta Mantovana, venne affidato a Vittorio Foschini, giornalista ma anche ex agente del cosiddetto servizio 6x, un servizio informativo attivato verso la fine del 1942 su iniziativa dello stesso Foschini, approvato prima dal Ministro della Cultura Popolare Buffarini Guidi, e in seguito dal Duce stesso al quale venivano indirizzate le "veline"41. In realtà, il Servizio era subordinato agli stessi tedeschi e quindi non poteva, secondo l'accordo stipulato tra Rodolfo Graziani e

37 TNA, WO 204/12441 Republican fascist intelligence organisation in Italy, rel. del 1 Maggio 1945 "Allied Force Headquarters counter espionage and counter sabotage summary for April 1945, Appendix A - The republican fascist intelligence service", p. 2. Aniceto Del Massa, fascista della prima ora, fu uno scrittore amico e collaboratore di Julius Evola. Nel dopoguerra diresse le pagine culturali del quotidiano del Movimento Sociale Italiano «Il Secolo d'Italia».

${ }_{38}$ CUCCO, Alfredo, Non volevamo perdere, Bologna, Cappelli, 1950, p. 117-118, cit. in CONTI, «La RSI e l'attività del fascismo clandestino nell'Italia liberata dal settembre 1943 all'aprile 1945", in Storia contemporanea, 10, 4-5/1979, pp. 941-1013, p. 954. Valerio Pignatelli, nato a Chieti nel 1886, era stato comandante degli Arditi nella Grande Guerra, aveva combattuto in Russia, Etiopia e Spagna, aveva aderito al fascismo ma era stato espulso fino a che nel 1943 Carlo Scorza lo nominò ispettore dei Fasci. Nel dopoguerra fu tra i fondatori del MSI. Vedi PARLATO, Giuseppe, Fascisti senza Mussolini. Le origini del neofascismo in Italia 1943-1948, Bologna, Il Mulino, 2006, p. 39.

39 CUCCO Alfredo, op. cit., p. 118.

40 AUSSME, SIM, b. 68, f. 1-1-7 Organizzazione e attività del SID, rel. "Servizio informazioni difesa", s.d., p. 1; PALA, Elena, op. cit., p. 160.

${ }^{41}$ VENTO, Andrea, In silenzio gioite e soffrite, Milano, Il Saggiatore, 2011, p. 282; ma anche AUSSME, SIM, b. 186, f. 1-1-7 Organizzazione e attività del SID, sottof. Documenti riguardanti il SID, lettera del 24 marzo 1943 di Vittorio Foschini a Benito Mussolini; Foschini venne però molto presto sostituito (gennaio 1944) dal colonnello dei Carabinieri Candeloro De Leo e addirittura internato dai tedeschi. Vedi PALA, Elena, op. cit., p. 162. 
Otto Hellferich, svolgere alcuna attività di spionaggio. L'organizzazione pertanto poteva dedicarsi esclusivamente all'attività di contro-spionaggio e contro-sabotaggio, nonché all'attività antipartigiana, in collaborazione con i tedeschi ${ }^{42}$.

I fascisti tuttavia non si accontentavano di operare secondo l'ordine tedesco o fornire uomini ai Servizi germanici. La loro azione era invero spesso disorganizzata o portata avanti dall'iniziativa di singoli gerarchi. Ad esempio possiamo citare il tentativo del sottosegretario alla Presidenza del Consiglio della RSI, Francesco Barracu, di organizzare un gruppo di persone di fiducia, capitanate da padre Luciano Usai, con lo scopo di costituire una rete informativa politico-militare e di propaganda in Sardegna, sua regione di origine ${ }^{43}$. Il gruppo venne addestrato e in seguito paracadutato nell'isola dai tedeschi, i quali inizialmente, come abbiamo visto, pur di avere personale a disposizione non si curavano più di tanto della lealtà degli agenti inviati in missione 44 . Anche questa iniziativa tuttavia fallì poiché il gruppo venne arrestato dalle autorità alleate 45 .

Altra iniziativa personale fu quella di Borghese il quale, nell'ottobre 1944, a causa del trattamento ritenuto inadeguato riservato ai suoi uomini da parte dei Servizi tedeschi (addestramento lacunoso, incuranza della sicurezza personale degli agenti), decise di ridurre la sua collaborazione e di istituire il battaglione Vega inquadrando in esso tutti gli elementi legati allo spionaggio e al sabotaggio e iniziando ad operare in maniera indipendente, limitandosi ad inviare rapporti mensili alle autorità germaniche ${ }^{46}$. La nuova unità venne costituita per raccogliere informazioni e compiere atti di sabotaggio nelle zone occupate dagli Alleati nonché di predisporre attività di spionaggio e sabotaggio nelle principali città dell'Italia settentrionale all'indomani dell'occupazione alleata 47 . Come spiega un documento redatto dall'Office of strategic services (OSS), il servizio segreto statunitense, le attività del Vega si svilupparono parallelamente a quelle «del servizio segreto germanico, del servizio segreto agli ordini di Pavolini [l'ufficio PdM, n.d.a.], e probabilmente del Mgir (Movimento giovani italiani

42 AUSSME, SIM, b. 68, f. 1-1-7 Organizzazione e attività del SID, rel. "Servizio informazioni difesa", s.d., p.1. In realtà esisteva una sezione Alfa addetta allo spionaggio ma come si evince dallo stesso documento la sua attività al di fuori dalla Repubblica è sempre stata impedita dai tedeschi. Ibidem, p. 4.

43 AUSSME, SIM, b. 27, f. 1-7-40 Lancio nelle campagne di Cabras a mezzo paracadute di presunti agenti nemici, Appunto del 27 luglio 1944, prot. n. 290/1944.

44 ACS, Allied Control Commission (d'ora in avanti ACC), Legal, f. 443 Case of Usai Luciano \& others (enemy agents, Sardinia), Report "Case of Usai Luciano and others", p. 1-4.

45 Ibidem.

46 National Archives and Record Administration, rg. 266, s. 108A, b. 254, f. Prince Valerio Borghese, Preliminay interrogation of Prince Valerio Borghese, cfr. TRANFAGLIA, Nicola, Come nasce la Repubblica. La mafia, il Vaticano e il neofascismo nei documenti americani e italiani, Milano, Bompiani, 2004, p. 13.

47 Ibidem. 
repubblicani)»48. Il Movimento giovani italiani repubblicani era un gruppo clandestino nato a Firenze nel 1943 il quale aveva l'obiettivo di eliminare i gerarchi tramite un colpo di Stato e restaurare la "purezza" del Fascismo. Secondo il controspionaggio alleato, essi avrebbero avuto contatti con Thun von Hohenstein (ma anche con la X Mas) il quale utilizzò il gruppo come serbatoio di spie da inviare oltre le linee, ben sapendo che in realtà il loro primo obiettivo era quello di mettersi in contatto con i membri del gruppo che si trovavano nel territorio occupato dagli Alleati 49.

Il quadro qui descritto mostra dunque un insieme intricato di gruppi e organizzazioni che avrebbero dovuto lavorare assieme al comune obiettivo di contrastare l'avanzata alleata ma che finivano per disperdere risorse umane e materiali preziosissimi. D’altronde il moltiplicarsi delle organizzazioni di spionaggio, rispecchiava pienamente il quadro della situazione e degli organismi operanti all'interno della Repubblica di Salò. Come scrive Luigi Ganapini, «[1]a molteplicità dei centri di potere e la varietà delle truppe riflette[va] un carattere di fondo della Repubblica, eversivo e velleitario, che ambi[va] a ripetere in forme nuove l'esperienza del fascismo del ventennio e a costruire un rinnovato ordine sul modello del totalitarismo nazista» $5^{0}$.

\section{I tentativi di riorganizzazione del fascismo nel Sud Italia}

Nonostante il fallimento dell'operazione pianificata da Barracu e la sostanziale immobilità del gruppo PdM, i tentativi di rivitalizzare il fascismo nel Sud Italia e nel contempo di istituire dei gruppi locali di sabotatori sembrarono dare frutti nel corso del 1944, quando iniziarono a svilupparsi i primi gruppi neofascisti nel territorio occupato dagli Alleati. Un documento del controspionaggio angloamericano ci aiuta a delineare le organizzazioni presenti in Puglia, Calabria e Campania51. Ancora una volta viene citato come leader dei gruppi resistenziali fascisti il Principe Pignatelli su indicazione di Barracu. I gruppi più numerosi e meglio sviluppati erano quelli capeggiati da Gaetano Chetry a Taranto, ex capo settore del PNF e dall'avvocato Luigi Filosa a Catanzaro ${ }^{52}$. I

\footnotetext{
48 Ibidem.

49 Vedi TNA, WO 204/12442 Movimento Gioventù Italiana Repubblicana (MGIR), rel. s.d., "Movimento Giovani Italiani Repubblicani (MGIR)".

50 GANAPINI, Luigi, op. cit., p. 13.

${ }^{1}$ AUSSME, SIM, b. 103, f. 1-7-1426 Sospetta attività di gruppi repubblicani fascisti nel Sud Italia, rel. del 19 novembre 1944 “Caso Mariani e gruppi repubblicani fascisti Sud Italia”, p. 1-2. $5^{2}$ Luigi Filosa, figura di fascista sui generis, nacque nel 1897 a Cosenza e fu tra i fondatori dei Fasci in Calabria. Venne espulso dal partito già nel 1923, dopo essere stato eletto federale, per la sua linea intransigente repubblicana e rivoluzionaria, avvicinandosi in seguito agli ambienti
} 
gruppi erano in contatto con l'Abwehr che aveva bisogno di assicurare una rete di supporto agli agenti paracadutati oltre le linee53. Filosa veniva indicato come il principale organizzatore della guerriglia alle spalle degli Alleati, in particolare nella provincia di Cosenza dove abitava e dove aveva predisposto, insieme ad un comitato di ex squadristi, l'invio di emissari nei diversi comuni per costituire dei nuclei fascisti54. I gruppi erano composti sia da vecchi fascisti che da studenti, uniti per la causa comune. Si fecero notare soprattutto per «svariati casi di dimostrazione contro gli Alleati e [...] attentati terroristici eseguiti con lo scopo di costringere la popolazione locale a sottomettersi e convincersi della forza e della volontà della causa fascista»55. Il movimento si era sviluppato in modo tale da permettere ai gruppi inizialmente isolati di concretare un'azione comune ${ }^{56}$. Era infatti attivo non solo in Calabria ma era presente anche in Puglia. A Bari il gruppo, nonostante gli arresti di molti aderenti, era riuscito a pubblicare anche un giornale denominato "Onore e combattimento", «notiziario di tutti coloro che ancora amano la Patria e sono pronti a servirla sino al sacrificio estremo»57. Pignatelli inoltre, tramite l'avvocato Nando di Nardo, teneva i collegamenti anche con i gruppi neofascisti presenti in Campania. I giovani in particolare si erano fatti sentire con proteste e lanci di manifestini contro gli Alleati ${ }^{8}$. Tra aprile e maggio 1944 i gruppi neofascisti vennero stroncati dalle forze dell'ordine le quali disposero una serie di arresti. In totale le persone portate a giudizio furono ottantotto, pertanto il processo venne denominato dalla stampa dell'epoca "processo degli ottantotto". Tra costoro, peraltro, non figurava, clamorosamente, il Principe Pignatelli, nonostante fosse stato indicato da tutti i componenti come il capo dell'organizzazione nel Sud Italia59. Lo stesso Pignatelli era stato arrestato, tuttavia, nell'aprile del ' 44 insieme alla moglie ed entrambi erano stati accusati di spionaggio. La principessa Maria, futura fondatrice del Movimento Italiano Femminile (MIF), aveva attraversato le linee su incarico del marito, con la scusa di visitare i figli residenti a

antifascisti. Dopo essere stato condannato a 3 anni di confino, nel febbraio del 1943 decise di rientrare nel PNF. Fu tra i fondatori del Movimento Sociale Italiano in Calabria e uno dei sei deputati eletti nelle liste missine nel 1948. Vedi MAZZA, Fulvio, «Filosa Luigi», in Dizionario Biografico degli italiani, vol. 48, Roma, Istituto dell'Enciclopedia Italiana, 1997, pp. 2-3.

53 AUSSME, SIM, b. 103, f. 1-7-1426 Sospetta attività di gruppi repubblicani fascisti nel Sud Italia, rel. del 19 novembre 1944 "Caso Mariani e gruppi repubblicani fascisti Sud Italia", p. 2.

54 ACS, DGPS, DAG, 1944-1946, b. 45, f. Partito fascista repubblicano Catanzaro vol. 1, rel. s.d., s.p. "Riorganizzazione fascista in Calabria", pp. 2-3.

55 Ibidem, p. 5 .

${ }^{56}$ Ibidem, p. 6.

57 ACS, DGPS, DAG, 1944-1946, b. 45, f. Partito fascista repubblicano Bari, "Onore e combattimento", anno I, n. I, 28 ottobre 1944, p. 1.

58 MAMMONE, Andrea, «The black-shirt resistance: Clandestine fascism in Italy, 1943-50», in The italianist, 27, 2/2007, pp. 282-303, p. 290.

59 PARLATO, Giuseppe, op. cit., pp. 56-59. 
Firenze, per incontrare Mussolini e Barracu ${ }^{60}$. Nel suo viaggio era stata aiutata da un agente del SIM impiegato nell'OSS, Paolo Poletti, che le aveva procurato i lasciapassare dell'amministrazione militare alleata ma al suo ritorno era stata arrestata dagli inglesi. Nonostante le gravi accuse, e le prove degli incontri con le personalità fasciste, i due Pignatelli vennero condannati solamente a pochi anni di carcere. Questa vicenda, che ha ancora molti lati oscuri, tra i quali la morte dello stesso Poletti (forse agente doppiogiochista a favore del governo fascista), mette in luce come esponenti di spicco del fascismo clandestino già nel corso del 1944 potessero contare su contatti con militari italiani e alleati ${ }^{61}$.

Dopo gli arresti del gruppo di Filosa e Chetry, il SIM su ordine degli Alleati, mise agli arresti gli appartenenti di piccoli gruppi a Bari, Lecce e in Sicilia ${ }^{62}$. Altri gruppi tuttavia erano ancora operanti in tutto il Sud Italia, secondo il rapporto dello stesso SIM, ed erano capeggiati da un certo Giorgio Bertacchini, agente dei servizi tedeschi, un'ulteriore dimostrazione dell'inevitabile stretto legame tra i gruppi fascisti e lo spionaggio nazista ${ }^{6}$.

\section{L'abbandono di Roma e le reti nazifasciste stay-behind nella capitale}

L'avanzata degli Alleati verso Roma e l'imminente abbandono della capitale italiana, rendeva necessario il trasferimento verso Nord degli uffici dell'apparato nazista compresi pertanto quelli addetti allo spionaggio. La sede romana del Primo e del Terzo Abwehr venne chiusa e il personale, compreso Hellferich, si spostò a Milano dove già era presente Hans Werner. La scuola di reclutamento di Parma venne spostata a Rovereto64. Il Secondo Abwehr si spostò invece a Bolzano così come la scuola di reclutamento in precedenza collocata a Perugia ${ }^{65}$. Anche il SD dovette chiudere la centrale romana. Karl Haas tuttavia non si trasferì nel quartier generale veronese ma si

6o Il MIF era un'associazione nata nel dopoguerra che svolgeva prevalentemente compiti assistenziali a favore dei fascisti ed ex internati. Per una ricostruzione delle sue attività, vedi BERTAGNA, Federica, «Un'organizzazione neofascista nell'Italia post bellica: il Movimento Italiano Femminile "Fede e Famiglia" di Maria Pignatelli di Cerchiara", in Rivista Calabrese di storia del '9oo, 9, 1/2013, pp. 5-32.

${ }^{61}$ Per approfondire vedi BERTAGNA, Federica, op. cit., pp. 62-69 ma anche ACS, ACC, Public Safety, f. 2613 "Pignatelli May 1944-June 1946".

62 AUSSME, SIM, b. 103, f. 1-7-1426 Sospetta attività di gruppi repubblicani fascisti nel Sud Italia, rel. del 19 novembre 1944, "Caso Mariani e gruppi repubblicani fascisti Sud Italia”, p. 5.

${ }^{63}$ Ibidem, pp. 1, 5 .

64 TNA, WO 204/12293, History of German intelligence organization in Italy 1943-1945, rel. s.d., "Appunti sull'organizzazione informativa tedesca in Italia", p. 35.

65 Ibidem, p. 50. 
fermò a Sant'Ilario d'Enza, nei pressi di Parma, dove divenne il direttore di una scuola di spionaggio denominata Einheit Ida che addestrava agenti da inviare nel territorio occupato dagli Alleati66. Il nuovo responsabile in Italia del servizio informativo delle SS divenne invece Otto Ragen, alias Begus. Il suo compito sarebbe stato quello di creare una serie di reti stay-behind fasciste e naziste, sulla falsariga del gruppo che lo stesso aveva organizzato in Grecia, per provare a contrastare l'avanzata anglo americana. Se dunque l'addestramento dell'Ida era finalizzato allo spionaggio, Begus organizzò a Campalto, presso Verona, una scuola che formasse principalmente sabotatori da inviare dietro le linee ${ }^{67}$. D’altronde la continua avanzata degli Alleati rendeva necessaria l'attività di sabotaggio per poter tentare di rallentare gli angloamericani. Lo stesso Abwehr, infatti, moltiplicò il numero delle sue scuole per spie e sabotatori. Nella stessa relazione, databile probabilmente novembre 1944, il SIM segnalava la presenza di altre due scuole del Primo Abwehr a Ponte Vigodarzere, nei pressi di Padova, e a Legnago, mentre il Secondo aveva spostato le proprie sedi in Trentino. Nel comune di Fai della Paganella veniva svolta l'istruzione preparatoria ai corsi di sabotaggio, mentre a Coredo venivano allenati nell'impiego degli esplosivi coloro i quali avessero superato la prima selezione. Infine gli addestrati venivano inviati a Dosson (in provincia di Treviso) dove si trovava il centro di raccolta dei sabotatori che attendevano di essere inviati in missione. Altre scuole minori si trovavano invece a Mortise (Padova), Desenzano sul Garda (Brescia) e Nogara (Verona) ${ }^{68}$.

Queste scuole, come le precedenti, reclutavano agenti da inviare oltre le linee tramite paracadute, oppure, e in numero maggiore, che attraversassero le linee a piedi. L'imminente ingresso a Roma da parte degli Alleati, tuttavia, offriva la possibilità di reclutare agenti da lasciare in incognito nella città con l'obiettivo di operare durante l'occupazione angloamericana. Il SD, in particolare, si adoperò per realizzare questo obiettivo, impiegando personale proveniente principalmente dall'ex milizia portuaria di Trieste e dai battaglioni speciali come la Muti69. Non era la sola: un gruppo di spie e sabotatori era stato approntato da Tommaso David (tenente colonnello della milizia ed ex squadrista) sempre in collaborazione con il servizio segreto tedesco, e dalla X Mas del Principe Borghese tramite il reparto sabotatori-nuotatori "Gamma”. Il gruppo di David, alle dipendenze dell'Abwehr, si era costituito a Roma in Piazza Colonna per poi

\footnotetext{
66 TNA, WO 204/12293, History of German intelligence organization in Italy 1943-1945, rel. s.d., "Appunti sull'organizzazione informativa tedesca in Italia", p. 35, p. 62.

67 Ibidem, p. 64.

${ }^{68}$ Ibidem, pp. 51-58.

${ }^{69}$ AUSSME, SIM, b. f. 1-19-2 Rete di sabotaggio che dovrebbe agire a Roma dopo la occupazione degli Alleati, rel. s.d., "Rete dei sabotatori".
} 
trasferirsi a Milano. La particolarità del suo gruppo di Sabotatori Attentatori, consisteva nella presenza di un sottogruppo composto esclusivamente da agenti femminili, il cosiddetto "Gruppo speciale A" di cui faceva parte anche Carla Costa, fervente fascista che a soli 17 anni entrò a far parte del gruppo David7o. La ragazza, il cui compito era quello di «raccogliere informazioni relative alla dislocazione dei comandi alleati, contrassegni dei veicoli, uniformi e distintivi, relazioni tra la popolazione civile e gli Alleati, morale della popolazione, situazione alimentare», riuscì a compiere tre missioni a Roma e Firenze prima di essere arrestata ${ }^{71}$. Il battaglione Gamma era invece una unità della Decima Mas, con sede a Valdagno in provincia di Vicenza, comandata dal tenente di vascello Eugenio Wolk e che godeva di una particolare autonomia in seno alla formazione di Borghese. Con l'imminente liberazione di Roma, Wolk organizzò una serie di agenti che rimanessero nella Capitale al fine di raccogliere informazioni nel momento in cui gli Alleati avrebbero occupato la città, per poi attraversare le linee e riferire al comando. Ad esempio possiamo riportare la testimonianza di Luigi Kalb il quale, successivamente catturato dagli Alleati, raccontò che ritornato a Valdagno, a lavoro ultimato, aveva steso una relazione contenente notizie sulla consistenza delle formazioni partigiane, sul movimento neofascista e sui mezzi e materiali a disposizione delle truppe alleate ${ }^{72}$.

\section{Gli ultimi mesi di guerra: la fine dell'Abwehr e l'Ufficio Pucci-Del Massa}

Oltre all'azione poco brillante e senza grandi risultati portata avanti dai servizi tedeschi, un'altra tegola si abbatté in seno allo spionaggio germanico: il coinvolgimento del capo dell'Abwehr, l'Ammiraglio Wilhelm Canaris, nel fallito attentato ad Hitler del 20 luglio 1944. Una delle conseguenze fu l'immediato scioglimento del servizio informativo della Wehrmacht e l'assorbimento dei suoi uomini e delle sue funzioni da parte del SD. Un cambiamento che però, a quanto sembra, non fu così immediato in Italia dove le due organizzazioni continuarono ad operare separatamente e senza cambiamenti nella linea di comando, almeno fino al gennaio $1945^{73}$.

$7^{7}$ CAIROLI, Roberta, Dalla parte del nemico. Ausiliarie, delatrici e spie nella Repubblica sociale italiana (1943-1945), Milano-Udine, Mimesis, 2013, p. 189-190.

${ }^{71}$ Ibidem, p. 196-197.

${ }^{72}$ USSME, SIM, b. 266, f.1-19-9/2 Gruppo Sabotatori-Nuotatori "Gamma", rel. del 12 giugno 1945, “Agente nemico Kalb Luigi, alias Kalbi Gino, alias Kalby Luigi”, n. prot. 5796.

73 TNA, WO 204/12879 German intelligence service in Italy, rel. del 9 febbraio 1945, "The German intelligence service in Italy", pp. 2-5. 
Come raccontato in precedenza, la fine dell'anno aveva visto un netto cambiamento anche nel campo dello spionaggio repubblicano con l'arrivo di Aniceto Del Massa ad affiancare Puccio Pucci. Secondo il controspionaggio statunitense, «Del Massa era la reale forza direttiva [dell'ufficio Pdm, n.d.a.] avendo avuto antecedentemente un'esperienza spionistica a Roma ed a Firenze»74. L'organizzazione era composta da gruppi di agenti ed individui isolati che avevano il compito di esercitare attività antipartigiana e di spionaggio politico nel territorio occupato dagli Alleati75. Tramite un certo capitano Bauer era in contatto con l'intelligence tedesca il cui aiuto era necessario per l'addestramento degli agenti e il loro invio nel territorio occupato dagli Alleati76. Secondo il contro-spionaggio alleato, il gruppo era diviso in diverse sezioni: tra le principali, una dedicata allo spionaggio, guidata direttamente da Del Massa e composta da una ventina di agenti; una composta da circa cinquanta sabotatori con il compito di agire alle spalle degli Alleati e in collaborazione con una terza, addetta alla guerriglia. Quest'ultima, guidata dal comandante della Brigata Nera Garibaldi Onorio Onori, era formata da circa 1500 persone ed aveva anche l'obiettivo di eliminare personalità alleate e pro Alleati77. L'interrogatorio dello stesso Del Massa permette di aggiungere alcuni elementi che approfondiscono l'organizzazione e i suoi compiti. In particolare, Del Massa aveva reclutato uomini e donne per due gruppi speciali: il primo, comandato da Carlo Dane, con il ruolo di comprare piccole aziende, preferibilmente negozi che sarebbero serviti come posti di adunata, dato impiego ai membri del gruppo e sarebbero stati sorgenti di entrata per il movimento fascista; il secondo comandato da Libero Pilotto la cui missione era quello di recuperare una stamperia e stampare piccoli giornali che avrebbero dovuto «diffondere la nuova ideologia italiana e fare appello non solo alla gioventù d'Italia ma alla gioventù d'Europa ${ }^{78}$. Avrebbe dovuto compiere una sottile propaganda e a poco a poco connettersi con i vecchi principi fascisti sotto una nuova forma. È interessante notare che sia il gruppo Libero che altri tre gruppi di sabotaggio, organizzati sempre da Del Massa, prendevano ordini da un certo Consignor agente del controspionaggio statunitense ${ }^{79}$. Il suo vero nome era in realtà Alfio

\footnotetext{
74 AUSSME, SIM, b. 212, f. 1-7-994 Organizzazione segreta fascista diretta da Pucci Puccio e del Massa Aniceto, rel. del 10 luglio 1945, "Organizzazione segreta fascisa diretta da Puccio Pucci e Aniceto del Massa”, n. prot. 101804/2/CS, p. 1.

75 Ibidem.

${ }^{76}$ TNA, WO 204/12441 Republican Fascist Intelligence Service, rel. del 1 Maggio 1945 "Allied Force Headquarters counter espionage and counter sabotage summary for April 1945”, p. 2.

77 TNA, WO 204/12441 Republican Fascist Intelligence Service, rel. del 1 Maggio 1945 "Allied Force Headquarters counter espionage and counter sabotage summary for April 1945", p. 2.

${ }^{78}$ AUSSME, SIM, b. 212, f. 1-7-994 Organizzazione segreta fascista diretta da Pucci Puccio e del Massa Aniceto, rel. del 6 settembre 1945, "Verbale di interrogatorio dettagliato di Aniceto del Massa", p. 7.

79 Ibidem, pp. 7-8.
} 
Campolmi, un partigiano delle brigate Giustizia e Libertà ingaggiato dagli angloamericani, il quale, assieme a Elena Franchetti, nome in codice "Arch", era riuscito ad infiltrarsi nell'organizzazione spionistica ${ }^{80}$. Inizialmente la coppia avrebbe dovuto infiltrarsi nel SD grazie anche alla conoscenza del tedesco da parte della Franchetti. Riuscirono in primo luogo a prendere contatti con gli esponenti del MGIR e quindi con l'organizzazione PdM conquistando la fiducia dello stesso Del Massa ${ }^{81}$. Consignor, nel marzo 1945, venne scelto dallo scrittore-spia per svolgere una missione nell'Italia invasa con l'obiettivo di raccogliere notizie sui fascisti presenti nella Firenze appena occupata dagli Alleati, localizzare la presenza dei campi di concentramento e provare a ottenere la lista dei fascisti ivi presenti, prendere contatto con il gruppo denominato «Italia e civiltà» (che pubblicava l'omonima rivista ed era formato tra gli altri da Ardengo Soffici e Barna Occhini), e infine raccogliere giornali e periodici pubblicati per provare a ricostruire il quadro della situazione dell'Italia alleata ${ }^{82}$. Mentre l'ex partigiano veniva inviato nella scuola di Karl Hass per l'addestramento, la Franchetti riusciva ad ottenere il posto da segretaria di Del Massa per aiutarlo a relazionarsi con il SD vista la sua ottima conoscenza del tedesco ${ }^{83}$. Un ruolo che le permetterà di copiare di nascosto i documenti del gruppo per poterli consegnare agli Alleati a guerra terminata ${ }^{84}$.

Il lavoro di Consignor permise inoltre di far conoscere agli Alleati la presenza di gruppi collegati all'Ufficio PdM ma che in realtà agivano direttamente su ordine di Pavolini, ovvero le cosiddette Brigate nere Italia invasa. L'obiettivo era quello di creare dei gruppi per ciascuna regione dell'Italia occupata dagli Alleati (Sardegna e Sicilia escluse) composti ognuno da circa 1500 persone con diversi compiti (propaganda

\footnotetext{
80 TNA, WO 204/12478 Consignor, rel. del 6 agosto 1945, "Operation Consignor". Si tratta di un caso di agente doppio, un "penetration agent» secondo le categorie britanniche, ovvero «a double [agent] who worked solely against other intelligence services to obtain information on their organisations, personnel, methods, and operations». Vedi KNOBELSPIESSE, A. V. «Masterman Revisited», in Studies in intelligence, 18, 1/1974, pp. 25-40, p. 30. L'articolo è consultabile all'URL: < https://www.cia.gov/library/center-for-the-study-of-intelligence/kentcsi/vol18no1/pdf/v18i1a02p.pdf > [consultato il 29 dicembre 2016]. Per altri casi di agenti doppi utilizzati dagli Alleati nel corso della Seconda Guerra Mondiale vedi CROWDY, Terry, Deceiving Hitler. Double cross and deception in World War II, Oxford, Osprey, 2008, ma anche FRANZINELLI, Mimmo, Guerra di spie. I servizi segreti fascisti, nazisti e alleati (1939-1943), Milano, Mondadori, pp. 83-99.

${ }^{81}$ TNA, WO 204/12478 Consignor, rel. del 5 aprile 1945, "Report on S.C.I. operation

Consignor", pp. 1-4.

82 Ibidem, pp. 5-6.

83 Ibidem, p. 6.

84 TNA, WO 204/12478 Consignor, rel. del 26 maggio 1945, "Conclusion of S.C.I. operation Consignor".
} 
politica, spionaggio, sabotaggio $)^{85}$. Il quartier generale politico e spionistico avrebbe dovuto trovarsi a Roma sotto il comando di Mario Scandone mentre la centrale per il sabotaggio a Firenze e guidata da Renzo Buggiani. Oltre agli agenti inviati oltre le linee con l'aiuto dei tedeschi, la maggior parte del personale avrebbe dovuto essere reclutata sul posto tra i gruppi di fascisti che si opponevano agli Alleati e al governo di Salerno. Questo piano, elaborato nei primi mesi del 1945, non fece tempo ad essere attuato. Scandone invece era riuscito, nel frattempo, a compiere tre missioni esplorative e riportare notizie direttamente a Mussolini sulla situazione nel Sud e nel Centro Italia, prima di essere catturato dagli Alleati ${ }^{86}$.

\section{Il fallimento dei servizi nazifascisti e il secondo dopoguerra}

Ormai la guerra si stava avviando verso la conclusione e con la sconfitta delle forze dell'Asse. Anche lo stesso SD aveva compreso la gravità della situazione e già nell'ottobre del 1944 il comandante della Sipo e del SD Wilhelm Harster aveva tentato di aprire un canale di trattativa con i servizi angloamericani tramite il presidente della SNIA Viscosa Franco Marinotti, ma senza successo ${ }^{87}$.

Come riporta un memorandum dell'intelligence italiana, l'unica opportunità rimasta per provare, almeno, ad impensierire gli Alleati era quella di provare a sfruttare la situazione instabile e i disordini nel territorio da loro occupato. Pertanto negli ultimi mesi di guerra il Servizio tedesco non si preoccupò più di provare ad evitare la cattura dei propri agenti non assegnandogli nemmeno più una storia e un nome di copertura. La storia di due agenti italiani, Salvatore Carotenuto e Luigi Locatelli, inizialmente membri dell'Abwehr e in seguito del SD è esemplificativa. Erano stati paracadutati a Napoli con l'obiettivo di sabotare depositi di carburante, organizzare un gruppo di sabotatori in loco, provare a contattare i gruppi fascisti locali, mettere in atto le misure necessarie al fine di pubblicare giornali clandestini e volantini propagandistici. Non era stata fornita loro né un nome né una storia di copertura e il loro ritorno al quartier generale del SD non era stato nemmeno tenuto in conto. Non stupisce dunque che

85 TNA, WO 204/11917 Fabbri Carlo, Scandone Mario, Individual case report, rel del 19 marzo 1945, "Interrogation report on Scandone Mario".

86 Ibidem.

87 AGA ROSSI, Elena, SMITH, Bradley F., La resa tedesca in Italia, Milano, Feltrinelli, 1980, p. 78. Nei mesi successivi il SD ritornò protagonista nel negoziato con gli Alleati avviato dall'Obergruppenführer delle SS Karl Wolff, noto come Operazione Sunrise, tramite il lavoro di Guido Zimmer, Klaus Huegel e Eugen Dollmann. Ibidem, p. 92-95. 
Carotenuto si fosse immediatamente arreso agli Alleati una volta sbarcato sulla costa napoletana, facendo inoltre arrestare il suo compagno di viaggio ${ }^{88}$.

Se i servizi tedeschi negli ultimi giorni di guerra avevano lasciato senza alcun ordine i propri agenti, come dimostra anche la testimonianza di Aldo Littardi, leader di un gruppo di spie e sabotatori stay-behind a Bologna, il quale decise di consegnarsi agli Alleati non avendo più ricevuto alcuna comunicazione da parte del SD, diversa fu la situazione in seno all'organizzazione fascista ${ }^{89}$.

Secondo una relazione che probabilmente si può far risalire al già citato Consignor, nei giorni immediatamente precedenti alla Liberazione, Pavolini, Del Massa e Pucci, decisero di destinare allo stesso Pucci ingenti somme di denaro sia in valuta italiana che estera in vista dell'imminente caduta del fascismo ${ }^{90}$. Lo stesso Del Massa il 25 aprile «faceva un forte rifornimento di soldi»91. Denaro che non poteva che servire al finanziamento delle organizzazioni del fascismo clandestino dopo la caduta di Salò. Fu lo stesso Pavolini infatti, come rivela una fiduciaria del 1946 alla divisione SIS del Ministero dell'Interno, a tracciare «i quadri della riorganizzazione clandestina del PNF [...] quando al Nord si ebbe la netta sensazione dell'imminente sconfitta»92. Si tratta di una relazione dettagliata ricca di informazioni sull'attività e sui componenti del neofascismo clandestino. L'organizzazione era diretta da un triumvirato composto da Olo Nunzi, già segretario particolare di Pavolini, dal gerarca Augusto Turati e dall'ultimo segretario del PNF Carlo Scorza. I tre risulteranno tra i fondatori del Movimento sociale italiano, così come altre personalità citate nella relazione: Ludovico Muratori, ex generale della Milizia a capo dell'organizzazione militare, Pino Romualdi ex vicepresidente del Pfr, indicato come a capo di bande paramilitari mentre Concetto Pettinato e Vanni Teodorani come membri della direzione93. Gli stessi Pignatelli e Borghese, nonostante fossero ancora detenuti, tenevano i contatti con il movimento neofascista, oltre a cercare di creare delle cellule fra i detenuti ${ }^{94}$. Non sono citati né i nomi di Del Massa né di Pucci ma apprendiamo che il figlio Italo era a capo dei neofascisti romani. Tuttavia è ipotizzabile che anche loro ne facessero parte, visto lo stretto legame con Pavolini. Una conferma ci viene dall'interrogatorio di tale Enzo

88 USSME, SIM, b. 334, f. 1-1-2/6 Studio sull'organizzazione del S.I. tedesco e repubblicano in Italia, rel. del 25 gennaio 1945, "Memoria circa l'organizzazione dei servizi informativi tedeschi", n. prot. 1229 , pp. $95-98$.

89 TNA WO204/12160 Littardi A. and others members of a Bologna espionage group of staybehind agents, rel. del 2 maggio 1945, "Interrogation report on Littardi Aldo", p. 3.

9o ACS, DGPS, SIS-II, b. 38, f. HP40 Penne stilografiche esplosive, rel. s.d., p. 1.

${ }^{91}$ Ibidem.

92 ACS, DGPS, SIS-II, b. 37, f. HP28 Attività fasciste, rel. del 18 agosto 1946, p. 1.

93 Ibidem, pp. 2-3.

94 Ibidem, p. 6. 
Bonciani, ex appartenente al gruppo PdM, il quale raccontò agli ufficiali del centro contro-spionaggio di Firenze del SIM di aver incontrato a Roma, nel maggio 1946, Pucci insieme a Romualdi e in un successivo incontro anche l'ex capitano dei Nuotatori Paracadutisti della Decima MAS Nino Buttazzoni. Bonciani era stato invitato entrambe le volte ad entrare nelle formazioni neofasciste denominate Squadre d'Azione Mussolini (SAM) guidate da ciascuno di loro95. Il primo incontro avvenne significativamente a casa di Mina Magri Fanti, futura collaboratrice della Principessa Pignatelli nel Movimento Italiano Femminile. Secondo le informazioni dello stesso Bonciani, anche Del Massa in quel periodo avrebbe risieduto a Roma dove avrebbe custodito «i fondi ingenti - per il finanziamento dell'organizzazione spionistica neofascista» ${ }^{96}$. Lo stesso Pucci inoltre, in un memoriale citato nel libro di Parlato, Fascisti senza Mussolini, datava l'inizio del suo rapporto con Romualdi già nel corso dell'avventura dell'ufficio PdM, organizzazione che, come anche le parole di Pucci confermano, «fu orientata verso lo scopo di tenere vivo lo spirito fascista e di aiutare le organizzazioni di carattere fascista che man mano nascevano nelle zone occupate dal nemico»97.

\section{Conclusione}

L’importanza delle strutture e delle azioni di intelligence nel corso del secondo conflitto mondiale è un dato ormai acquisito anche dalla storiografia italiana, più restia rispetto a quella anglosassone ad occuparsi di storia dello spionaggio ${ }^{98}$. La stretta collaborazione dei servizi alleati di supporto all'esercito, nonostante non mancasse una forte rivalità e diffidenza tra l'OSS americano e lo Special Operation Executive (SOE) britannico, nonché, per quanto riguarda il caso italiano, anche con il SIM cobelligerante, fu uno dei fattori che contribuirono alla vittoria alleata ${ }^{99}$. Per quanto riguarda lo spionaggio nazista invece, emerge, anche da questa breve analisi delle sue strutture, la scarsa collaborazione, la rivalità e il danneggiamento a vicenda tra Abwehr

95 AUSSME, SIM, b. 432, f. 2-6-70 Bonciani Enzo, rel. del 31 ottobre 1946, "Bonciani Enzo", n. prot. 18084 , p. 1.

96 AUSSME, SIM, b. 432, f. 2-6-70 Bonciani Enzo, rel. del 31 ottobre 1946, "Bonciani Enzo", n. prot. 18084 , p. 2.

97 Memoriale di Puccio Pucci, cit. in PARLATO, Giuseppe, op. cit., p. 405.

${ }^{8}$ MASSIGNANI, Alessandro, FERRARI, Paolo, op. cit., pp. 15-18.

99 Per i rapporti tra OSS e SOE in Italia vedi STAFFORD, David, Mission accomplished. SOE and Italy 1943-1945, London, Bodley Head, 2011, pp. 32-34; WOODS, Christopher, SOE in Italy, in SEAMAN, Mark (edited by), Special Operation Executive. A new instrument of war, London-New York, Routledge, 2006, pp. 100-101; SAPIO, Michaela, op. cit., pp. 13-14. Per i rapporti tra intelligence britannica e statunitense nel corso della Seconda Guerra Mondiale vedi JAKUB, Jay, Spies and saboteurs:Anglo-american collaboration and rivalry in Human intelligence collection and Special operations (1940-1945), New York, St. Martin's Press, 1999. 
e SD almeno fino all'inizio del 1945, quando ormai però la guerra era compromessa. In Italia la situazione era ancora più complessa per la presenza del fascismo repubblicano e della volontà dei diversi gerarchi di giocare un ruolo attivo anche nel settore spionistico. Un'attività impossibile da realizzare senza l'aiuto dei tedeschi, i quali, come abbiamo visto, disponevano di uomini di collegamento tra i loro servizi e le organizzazioni fasciste. Gli agenti della RSI spesso però, una volta varcate le linee, agivano in autonomia e con obiettivi propri. Un'altra grande differenza tra Alleati e Tedeschi era ovviamente la scelta dei secondi di adoperare quasi esclusivamente agenti reclutati in loco la cui affidabilità, nonostante l'addestramento meticoloso, era quanto meno dubbia.

Le organizzazioni spionistiche fasciste rispecchiavano la fragilità e la mancanza di coordinamento delle forze della Repubblica Sociale, sprecando in questo modo uomini e mezzi che avrebbero potuto essere utili alla causa. Tuttavia, nonostante la loro debolezza, furono in grado di perseguire con successo il loro secondo obiettivo (il primo era ovviamente contrastare l'avanzata Alleata e vincere la guerra), ovvero la sopravvivenza dell'ideale fascista. Sebbene in numero sicuramente minore di quanto avessero sperato, gli ex fascisti repubblicani e tra loro molti di coloro i quali avevano partecipato ai servizi di spionaggio, già nella seconda metà del 1945 erano in grado di aggregarsi e svolgere attività di propaganda nelle diverse sigle del fascismo clandestino $^{100}$. La loro libertà di azione venne favorita soprattutto dalla fallita opera di epurazione e dall'amnistia Togliatti promulgata l'anno successivo ${ }^{101}$. Come scrive Andrea Mammone, «[t]he 1946 political amnesty granted to fascists, represented a sort of political and social legitimization for the former black-shirt enemies. These efforts at

\footnotetext{
100 MAMMONE, Andrea, op. cit., pp. 290-293; PARLATO, Giuseppe, op. cit., pp. 156-169, 211238.

${ }^{101}$ Un caso clamoroso fu ad esempio, quello di Borghese. Prosciolto dalla sezione istruttoria del Tribunale di Roma dall'accusa omicidio per aver fatto fucilare 43 partigiani dai reparti ai suoi ordini, venne rinviato a giudizio per collaborazionismo e per concorso in un numero limitato di omicidi. La Corte di Assise di Roma nel febbraio 1949 lo condannò a dodici anni di reclusione. Tuttavia «dopo aver letto il dispositivo della sentenza, il presidente della corte si accorse che il conteggio degli anni di reclusione era errato. Infatti, ai sensi della legge del 1946, il condono avrebbe dovuto essere maggiore di un anno rispetto a quello calcolato dalla corte, il che avrebbe consentito a Borghese, che aveva scontato già un certo periodo di carcerazione preventiva, di uscire immediatamente dal carcere. In violazione di ogni principio di procedura [...], il presidente della corte riportò immediatamente i componenti di questa in camera di consiglio, dove provvide a rettificare la misura del condono [...]. Borghese ritornò in questo modo in libertà». Vedi CANOSA, Romano, Storia dell'epurazione in Italia. Le sanzioni contro il fascismo (1943-1948), Milano, Baldini\&Castoldi, 1999, pp. 366-369. Per quanto riguarda l'epurazione nel nostro Paese di diverso avviso invece WOLLER, Hans, I conti con il fascismo. L'epurazione in Italia (1943-1948), Bologna, Il Mulino, 1997. Per un recente studio sulla "giustizia di transizione" vedi FOCARDI, Giovanni, NUBOLA, Cecilia (a cura di), Nei tribunali. Pratiche e protagonisti della giustizia di transizione nell'Italia repubblicana, Bologna, Il Mulino, 2015.
} 
national reconciliation could not serve to divide the extreme right but instead gave it, even if unintentionally, a new vigour» ${ }^{102}$.

Nel dicembre 1946, a poco più di un anno dalla fine della guerra, i diversi gruppi del fascismo clandestino sfruttarono l'opportunità riuscendo a riunirsi sotto l'ombrello di un nuovo partito politico legalmente riconosciuto, il Movimento Sociale Italiano ${ }^{103}$.

${ }_{102}$ MAMMONE, Andrea, op. cit., p. 297. Per uno studio sugli effetti dell'amnistia Togliatti, e la sua applicazione da parte della Corte di Cassazione, vedi FRANZINELLI, Mimmo, L'amnistia Togliatti. 22 giugno 1946, colpo di spugna sui crimini fascisti, Milano, Mondadori, 2006.

103 PARLATO, Giuseppe, op. cit. , pp. 238-254. 


\section{* L'autore}

Nicola Tonietto ha conseguito la laurea magistrale in Scienze Storiche presso l'Università di Padova nel 2014. È attualmente dottorando dell'Università di Trieste e Udine con un progetto di ricerca sulle origini del movimento neofascista in Italia.

URL: < http://www.studistorici.com/progett/autori/\#Tonietto >

\section{Per citare questo articolo:}

TONIETTO, Nicola, "Le reti di spionaggio e sabotaggio nazifasciste nell'Italia occupata dagli Alleati (1943-1945)», Diacronie. Studi di Storia Contemporanea : La voce del silenzio: intelligence, spionaggio e conflitto nel XX secolo, 29/12/2016,

URL:< http://www.studistorici.com/2016/12/29/tonietto_numero_28/ >

Diacronie Studi di Storia Contemporanea $\beta$ www.diacronie.it

Risorsa digitale indipendente a carattere storiografico. Uscita trimestrale. redazione.diacronie@hotmail.it

Comitato di redazione: Jacopo Bassi - Luca Bufarale - Antonio César Moreno Cantano - Deborah Paci - Fausto Pietrancosta - Alessandro Salvador - Matteo Tomasoni - Luca Zuccolo

Diritti: gli articoli di Diacronie. Studi di Storia Contemporanea sono pubblicati sotto licenza Creative Commons 3.0. Possono essere riprodotti e modificati a patto di indicare eventuali modifiche dei contenuti, di riconoscere la paternità dell'opera e di condividerla allo stesso modo. La citazione di estratti è comunque sempre autorizzata, nei limiti previsti dalla legge. 